\title{
Influence of GSTM1 and GSTT1 polymorphisms on the survival rate of patients with malignant glioma under perillyl alcohol-based therapy
}

\author{
M.M. Silva ${ }^{1}$, C.O. Da Fonseca ${ }^{2}$, R. Moura-Neto ${ }^{3}$, J.F. Carvalho ${ }^{4}$, \\ T. Quirico-Santos ${ }^{5}$ and M.G. Carvalho ${ }^{1}$ \\ ${ }^{1}$ Laboratório de Controle da Expressão Gênica, Instituto de Biofísica, \\ Universidade Federal do Rio de Janeiro, Rio de Janeiro, RJ, Brasil \\ ${ }^{2}$ Departamento de Medicina Geral e Especializada, Faculdade de Medicina, \\ Universidade Federal Fluminense, Niterói, RJ, Brasil \\ ${ }^{3}$ Instituto de Biologia, Universidade Federal do Rio de Janeiro, \\ Rio de Janeiro, RJ, Brasil \\ ${ }^{4}$ Instituto Carlos Chagas, FIOCRUZ, Curitiba, PR, Brasil \\ ${ }^{5}$ Laboratório de Patologia Celular, Departamento de Biologia Celular e Molecular, \\ Instituto de Biologia, Universidade Federal Fluminense, Niterói, RJ, Brasil
}

Corresponding author: T. Quirico-Santos

E-mail: tquirico@vm.uff.br

Genet. Mol. Res. 12 (2): 1621-1630 (2013)

Received May 28, 2012

Accepted November 30, 2012

Published May 14, 2013

DOI http://dx.doi.org/10.4238/2013.May.14.2

\begin{abstract}
GSTM1 (glutathione $S$-transferase mu 1) and GSTT1 (glutathione $S$-transferase theta 1 ) are critical enzymes for detoxification of endogenous and environmental carcinogens. Constitutive GST gene polymorphisms may be associated with increased risk for cancer development. We made an explorative study of a Brazilian population with malignant glioma to determine whether GSTM1 and GSTT1 genetic polymorphisms influence the response to intranasal administration of perillyl alcohol and the survival rate. Patients were stratified into groups according to clinical presentation, tumor classification, and tumor location. Circulating DNA was extracted from blood plasma or serum, and genotypes were detected by multiplex PCR. The cohort included
\end{abstract}


95 patients with recurrent malignant glioma included in a Phase I/II clinical trial with perillyl alcohol and 100 matched healthy control subjects. GSTM1 frequency was similar in patients with glioma (44\%) and healthy controls (54\%), but GSTT1 deletion was found in $11.5 \%$ patients, contrasting with $36 \%$ in controls. A longer survival rate was associated with a lack of GSTM1 deletion (31 weeks) and a deletion for GSTT1 (28 weeks). A poor survival rate was associated with GSTM1 deletion (23 weeks) and with a lack of a GSTT1 deletion (19 weeks). A significantly lower frequency of GSTT1 deletion in glioma patients compared to healthy controls indicates that GSTT1 deletion may exert a protective role against gliomagenesis, influence therapeutic response to intranasal perillyl alcohol treatment, and increase overall survival, especially considering tumor topography.

Key words: Glutathione $S$-transferases; Genetic polymorphism; Glioma; Perillyl alcohol

\section{INTRODUCTION}

Gliomas are the most frequent, heterogeneous group of primary malignant tumors in the adult central nervous system, accounting for more than $50 \%$ of all brain tumors (Louis et al., 2007; Adamson et al., 2010). Patients with malignant glioma generally have poor prognosis with 5 -year survival less than 5\% due to tumor aggressiveness and lack of curative treatment (Adamson et al., 2010). In addition, the anatomical location of the lesion in the brain may affect the rate of tumor growth, with gliomas developing slowly in the deep gray matter but showing increased proliferation in the lobar white matter (Ramnarayan et al., 2007; Adamson et al., 2010).

Environmental/occupational exposure and DNA damage are potential neurocarcinogenic factors (De Roos et al., 2003; Pinarbasi et al., 2005), which increases the importance of glutathione $S$-transferase (GST) systems for detoxification of exogenous and endogenous substances (Pinarbasi et al., 2005; Belpomme et al., 2007). Interestingly, under similar conditions, individuals respond differently to environmental carcinogen exposure and present distinct genetic susceptibility to cancer; the age-adjusted incidence of brain tumors is high in developed industrial countries (Ohgaki and Kleihues, 2005).

The human GSTs possess enzymatic and non-enzymatic functions and are involved in phase II metabolism, stress response, cell proliferation, apoptosis, oncogenesis, tumor progression, and drug resistance (Lo and Ali-Osman, 2007). GST isoenzymes catalyze the conjugation of reactive electrophiles generated during phase I detoxification and scavenging of free radicals created by radiation (Kilburn et al., 2010) forming water-soluble compounds easily removed by the body, including polycyclic aromatic hydrocarbon carcinogens (Pinarbasi et al., 2005; Belpomme et al., 2007; Kilburn et al., 2010). Based on sequence homology and immunological cross-reactivity, human GSTs have been grouped into 8 families: alpha, mu, theta, pi, zeta, sigma, kappa, and omega (Hayes et al., 2005).

Homozygous deletion of GSTM1 and GSTT1 genes eliminate GSTM1 and GSTT1 functional enzyme production (Hayes et al., 2005; Pinarbasi et al., 2005). A high percentage of American Caucasians (50\%) possess the GSTM1 null genotype, whereas only 15\% lack 
GSTT1, which has been associated with increased susceptibility to meningioma (De Roos et al., 2003; Lai et al., 2005; Pinarbasi et al., 2005; Schwartzbaum et al., 2007) but not esophageal cancer in Whites and Asians; neither is associated with esophageal squamous cell carcinoma in Brazilian subjects (Lee et al., 2005). In contrast, lack of GSTM1 is associated in some groups with increased risk of solid tumors in the colon (Ebrahimkhani et al., 2012); bladder (Engel et al., 2002), lung (Carlsten et al., 2008), and stomach (Chen et al., 2010; Qiu et al., 2011). Studies correlating GST polymorphisms and risk of adult brain tumor have produced inconsistent results, indicating the need to establish a putative association with malignant gliomas, the most frequent and aggressive adult brain tumor.

Perillyl alcohol (POH), a dietary monoterpene found in a variety of plants, suppresses post-translational isoprenylation of the Ras small GTPase superfamily of proteins that stimulate tumor-associated angiogenesis and NFKB signaling (Holstein and Hohl, 2003; da Fonseca et al., 2008a; Chaudhary et al., 2009). This study compared the genotypic profile of GSTM1 and GSTT1 in a group of Brazilian patients with malignant glioma and a control group of healthy individuals living in the same geographical region. The aim was to establish whether GSTM1 and GSTT1 polymorphisms influence survival and response to intranasal administration of $\mathrm{POH}$.

\section{MATERIAL AND METHODS}

This case-control study included a group of patients with malignant gliomas enrolled in a Phase I/II clinical trial to assess the efficacy of intranasal administration of the monoterpene $\mathrm{POH}$. The cohort consisted of 95 patients (52 men and 43 women) with recurrent malignant glioma, mean age 53.4 years (range 19 to 83). Diagnosis and histological classification of malignant glioma based upon WHO criteria (Louis et al., 2007) indicated the following distribution: glioblastoma multiforme ( $\mathrm{GBM} ; \mathrm{N}=71)$, anaplastic astrocytoma (AA; $\mathrm{N}=13$ ) and oligodendroglioma $(\mathrm{OD} ; \mathrm{N}=11)$. All patients were attending the outpatient Neurosurgical Unit of Antonio Pedro University Hospital. The control subjects included 100 healthy volunteers ( 36 men and 64 women), mean age 42.6 years (range 20 to 97). Demographic characteristics of patients and controls are shown in Table 1. Control subject eligibility was assessed by a detailed questionnaire; only subjects with no familial history of brain tumor, infectious disease, or use of anti-inflammatory medication were included. The Hospital Medical Research Ethics Committee and the Brazilian Ministry of Health (CONEP 25000.009267/2004-25) approved the study, which complies with the principles laid down in the Declaration of Helsinki.

\begin{tabular}{|c|c|c|}
\hline Demographic characteristics & Control subjects $(\mathrm{N}=100)$ & Glioma patients $(\mathrm{N}=95)$ \\
\hline Male (\%) & $36(36 \%)$ & $52(55 \%)$ \\
\hline Female (\%) & $64(64 \%)$ & $43(45 \%)$ \\
\hline Mean age (range) years & $42.6(20-97)$ & $53.4(19-83)$ \\
\hline Glioblastoma (\%) & 0 & $71(75 \%)$ \\
\hline Astrocytoma (\%) & 0 & $13(14 \%)$ \\
\hline Oligodendroglioma (\%) & 0 & $11(11 \%)$ \\
\hline
\end{tabular}

Patients were stratified into groups according to clinical presentation (primary or secondary GBM), classification (GBM, AA, OD), and tumor location (deep gray matter or lobar regions) confirmed by computer tomography and/or magnetic resonance imaging. Patients re- 
ceived $55 \mathrm{mg}$ POH 4 times daily by intranasal administration (inhalation) for a total dose of 220 $\mathrm{mg}$ /day. Intranasal delivery is a suitable route for efficient and rapid delivery of small molecules with molecular weight less than $1 \mathrm{kDa}$ (McMartin et al., 1987). The monoterpene $\mathrm{POH}$ is 152.8 $\mathrm{Da}$ and is hydrophobic, which allows it to cross the blood-brain barrier and reach the central nervous system, eliminating systemic losses and reducing side effects (da Fonseca et al., 2008b).

Circulating DNA was extracted fromblood plasma or serum(Fleischhacker and Schmidt, 2007; Cabral et al., 2010) with the QIAamp DNA Blood ${ }^{\circledR}$ kit according to manufacturer protocols. Control DNA samples were extracted from cells from the oral mucosa and treated with 0.3 $\mu \mathrm{g} / \mu \mathrm{L}$ proteinase $\mathrm{K}$ at $60^{\circ} \mathrm{C}$ for $2 \mathrm{~h}$. Thereafter, an equal volume of $1: 1(\mathrm{v} / \mathrm{v})$ phenol:chloroform was added, followed by vigorous shaking and centrifugation. The aqueous phase was separated and DNA precipitated with 2 volumes of absolute ethanol at $-20^{\circ} \mathrm{C}$ overnight. After centrifugation, pellets were washed with $70 \%$ ethanol and the DNA was resuspended in Milli-Q water. GSTM1 and GSTT1 genotypes were detected by multiplex polymerase chain reaction (PCR) with the $p 53$ gene (exon 5) as an internal control. The following primers were used: GSTM1, 5'-GAACTCCCTGAAAAGCTAAAGC-3' and 5'-GTTGGGCTCAAATATACGGTGG-3'; GSTT1, 5'-TTCCTTACTGGTCCTCACATCTC-3' and 5'-TCACCGGATCATGGCCAGCA3'; p53 (exon 5), 5'-GCAACCAGCCCTGTCGTGTCTCCA-3' and 5'-GGAATTCTGTTCA CTTGTGCCCTGACTTTCAAC-3'. The amplification conditions included denaturation at $94^{\circ} \mathrm{C}$ for $5 \mathrm{~min}$ followed by 30 cycles of $95^{\circ} \mathrm{C}$ for $30 \mathrm{~s}, 64^{\circ} \mathrm{C}$ for $1 \mathrm{~min}, 72^{\circ} \mathrm{C}$ for $1 \mathrm{~min}$. The final extension was at $72^{\circ} \mathrm{C}$ for $5 \mathrm{~min}$. After amplification, PCR products were separated by $10 \%$ polyacrylamide gel electrophoresis and visualized with silver nitrate. Gel staining was performed as follows: an initial step of DNA fixation using a $10 \%$ ethanol and $0.37 \%$ acetic acid solution for $10 \mathrm{~min}$, a step of impregnation by $0.2 \%$ silver nitrate solution for $10 \mathrm{~min}$ followed by a rinse with distilled water for $30 \mathrm{~s}$, and a final step with $3 \% \mathrm{NaOH}$ and $0.4 \%$ formaldehyde solution until the DNA bands were clearly visible. The initial solution was used to stop the reaction. The SPSS 15.0 software was used for data analysis. The two-tailed Fisher exact test and odds ratio were used to compare groups (patients and control subjects) in the context of GSTM1 and GSTT1 deletion, and to determine the association between GSTM1 and GSTT1 genotypes with age, gender, and tumor type. The Williams G-test for independent samples was used to assess the correlation between GSTM1 and GSTT1 genotypes and median survival, and to correlate tumor location with survival. The Kaplan-Meier curves were used to analyze the association of GSTM1/GSTT1 genotypes and tumor location with survival.

\section{RESULTS}

Subjects (95 patients with malignant glioma and 100 controls) were genotyped for GSTM1 and GSTT1. The mean ages of patients and controls were $53.36 \pm 16.0$ and $42.6 \pm$ 20.2 , respectively. There was no statistically significant difference in familial cancer history between groups. Seventy-one glioma patients $(75 \%)$ had at least 1 clinical and image recurrence event from primary and $24(25 \%)$ from secondary GBM. Mean age significantly differed $(\mathrm{P}<0.01)$ between AA $(41.4 \pm 16.62)$ and primary GBM $(55.03 \pm 15.07)$, but not anaplastic OD $(52.4 \pm 5.51)$, confirmed by the Dunn multiple comparison test.

A representative gel image (Figure 1) shows GSTM1 and GSTT1 genes as 220- and 450-bp bands; $p 53$ (exon 5) appears as a 274-bp band. Genotype frequencies are shown in Table 2. GSTM1 deletion occurred in $42(44 \%)$ patients and $54(54 \%)$ control subjects $(\mathrm{P}=$ 
0.19). GSTT1 deletion was detected in $11(11.5 \%)$ patients and $36(36 \%)$ control subjects, a statistically significant difference: $\mathrm{OR}=0.23 ; 95 \% \mathrm{CI}=0.11-0.49 ; \mathrm{P}=0.00009$. Genotype frequencies stratified by histology revealed a high GSTT1 frequency in patients with primary GBM $(91.5 \%)$ and secondary GBM derived from anaplastic OD $(90.9 \%)$, but not in those with AA (69.2\%). Patients with AA carried the GSTM1 (76.9\%) allele more frequently than those with primary GBM $(50.7 \%)$ or anaplastic OD $(63.6 \%)$.

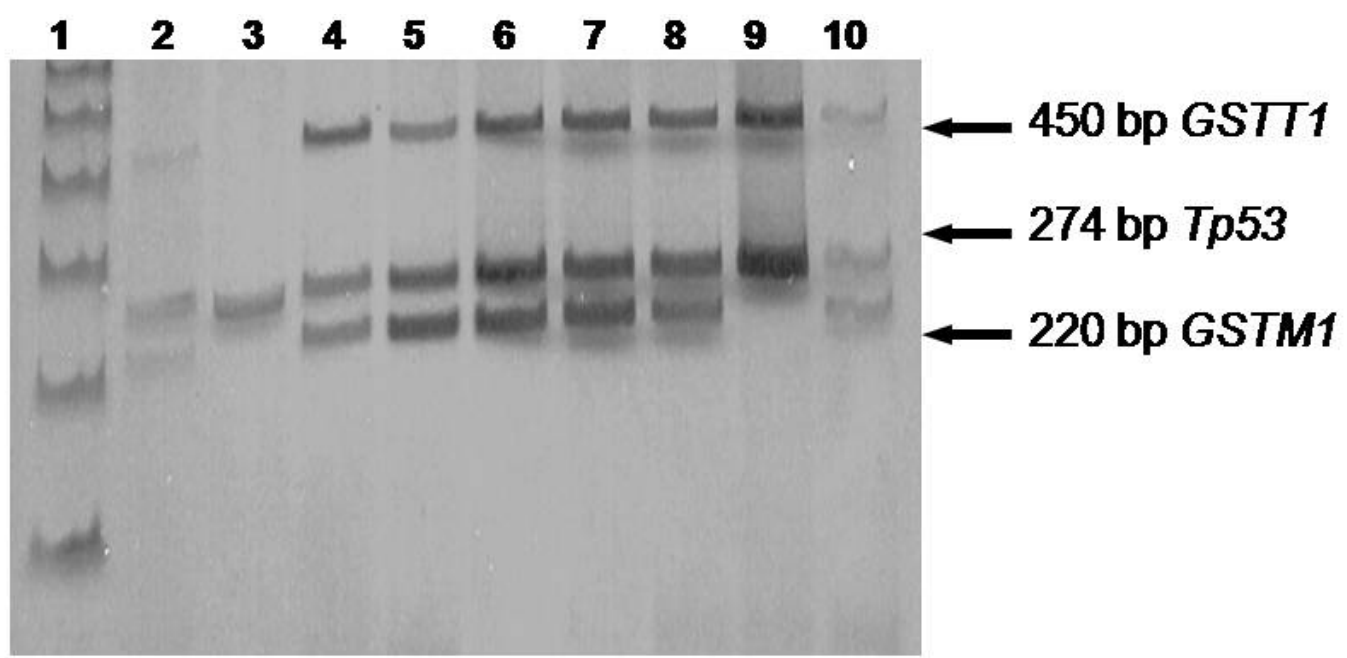

Figure 1. Multiplex PCR of GSTM1 and GSTT1 genes and Tp53 exon 5. Representative electrophoresis shows GSTM1 and GSTT1 genes as 220- and 450-bp bands, respectively, and p53 (exon 5) as a 274-bp band. Lane $1=$ 100-bp ladder; lanes 2, 4, 5, 6, 7, 8, and 10 = without deletion of GSTT1 or GSTM1; lane 3= GSTT1 and GSTM1 deletion; lane $9=$ GSTM1 deletion.

Table 2. Frequencies of GSTM1 and GSTT1 polymorphisms in patients and controls.

\begin{tabular}{lcc}
\hline Genotype & Control subjects $(\mathrm{N}=100)$ & Glioma patients $(\mathrm{N}=95)$ \\
\hline GSTM1 present (\%) & $46(46 \%)$ & $53(56 \%)$ \\
GSTM1 nul (\%) & $54(54 \%)$ & $42(44 \%)^{*}$ \\
Odds ratio (95\%CI) & 1.00 & $0.67(0.38-1.18)$ \\
GSTM1 present (\%) & $64(64 \%)$ & $84(88 \%)$ \\
GSTM1 nul (\%) & $36(36 \%)$ & $11(11.5 \%) * *$ \\
Odds ratio (95\%CI) & 1.00 & $0.23(0.11-0.49)$ \\
GSTM1/T1 present $(\%)$ & $30(30 \%)$ & $45(47 \%)$ \\
GSTM1/T1 nul (\%) & $20(20 \%)$ & $3(3 \%)^{* * *}$ \\
Odds ratio (95\%CI) & 1.00 & $0.10(0.02-0.36)$ \\
\hline
\end{tabular}

$* \mathrm{P}=$ not significant, $* * \mathrm{P}=0.00009, * * * \mathrm{P}=0.000085$

Survival by GSTM1 and GSTT1 genotypes was determined by Kaplan-Meier curves, shown in Figure 2 (Lai et al., 2005; Kilburn et al., 2010). Patients without GSTM1 but with the GSTT1 deletion had a greater survival rate; patients with GSTM1 deletion had a median survival of 23 weeks, and those without the deletion survived 31.3 weeks $(\mathrm{P}=0.86)$. Patients with the GSTT1 deletion presented a median survival of 18.7 weeks in comparison with 28.4 weeks in 
those without the deletion $(\mathrm{P}=0.0001)$. The influence of anatomical tumor location on survival rate was also investigated (Figure 3). Tumor location was available for 44 patients; of these, 29 had lobar tumors with median survival of 33 weeks. Patients with tumoral lesions in deep regions $(15 / 44)$ had a median survival of 39 weeks, a statistically significant difference $(\mathrm{P}=0.03)$.
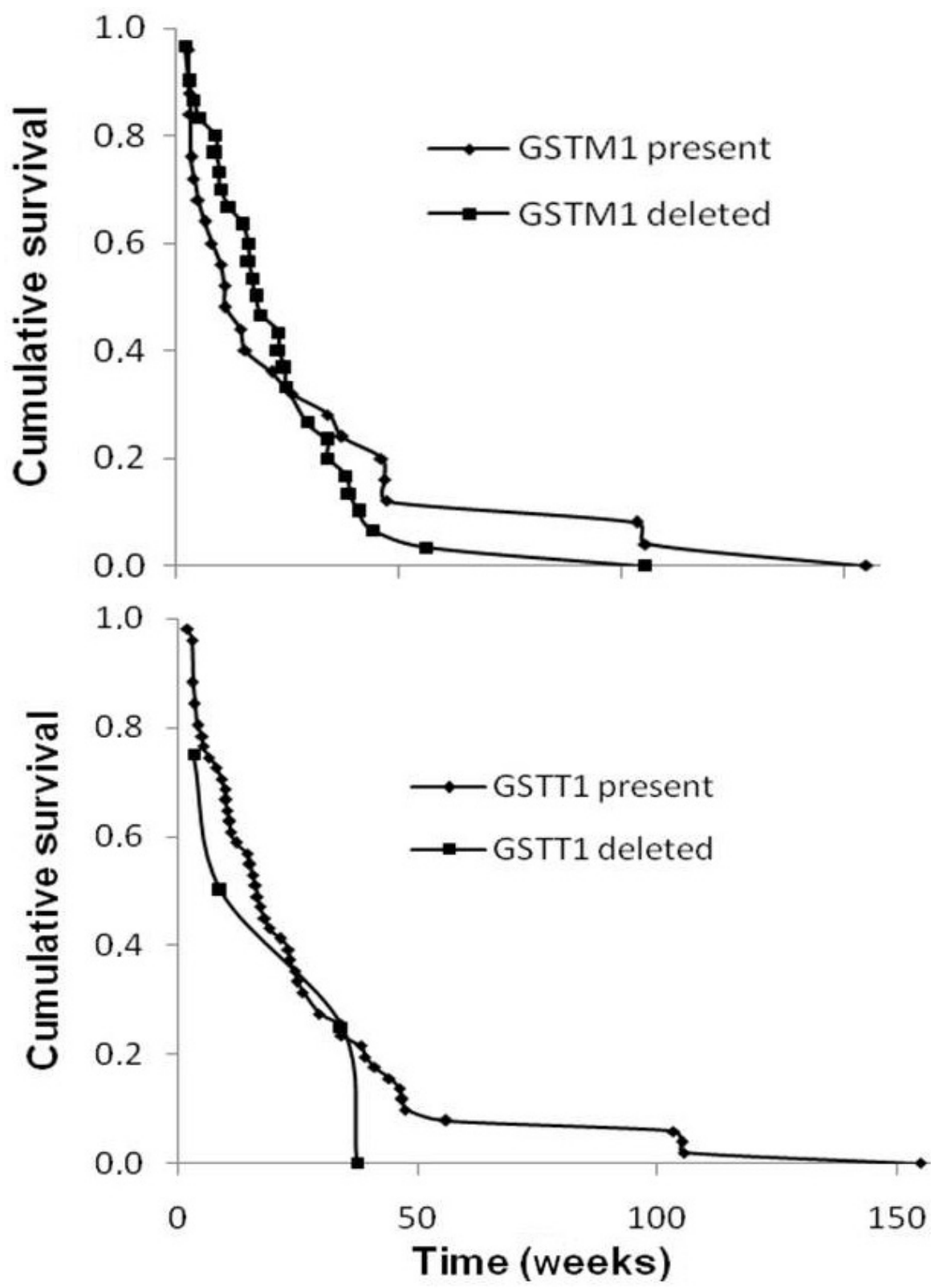

Figure 2. Survival of glioma patients under POH treatment stratified by genotypes. The Kaplan-Meier graphs show survival rates of patients according to GSTM1 and GSTT1 genotypes. 


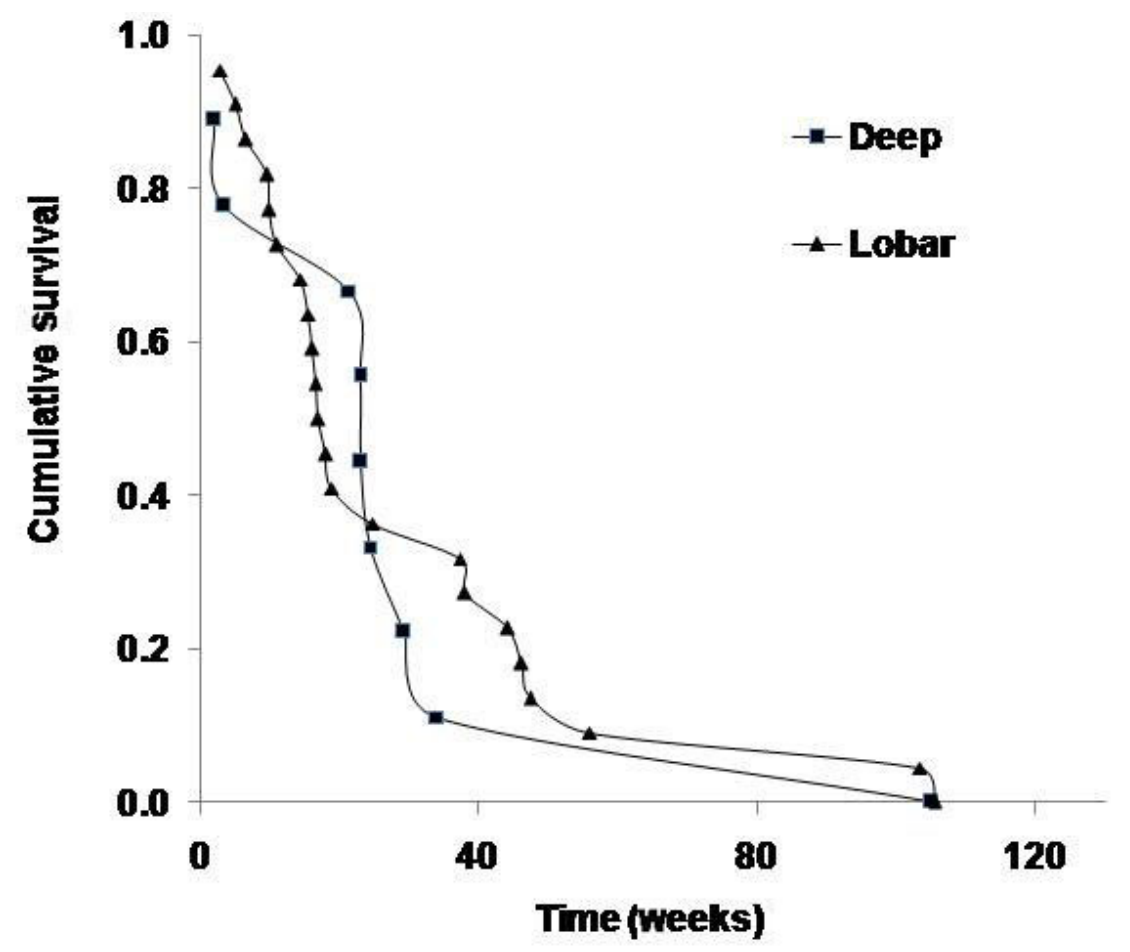

Figure 3. Survival of glioma patients under $\mathrm{POH}$ treatment stratified by tumor topography. The Kaplan-Meier graph shows survival rate of patients $(\mathrm{N}=44)$ according to anatomical tumor location. Patients with tumoral lesion in the lobar region $(\mathrm{N}=29)$ had median survival of 33 weeks and patients $(\mathrm{N}=15)$ with tumoral lesion in deep regions had median survival of 39 weeks $(\mathrm{P}=0.03)$.

\section{DISCUSSION}

Malignant gliomas are biologically aggressive brain tumors associated with high morbidity and mortality. A meta-analysis of publications from different populations including 1630 cases of glioma and 7151 controls did not find an association between GSTM1, GSTT1, and GSTP 1 variants and the risk of glioma (Lai et al., 2005; Kilburn et al., 2010). Moreover, GSTs show substantial variations in frequencies between ethnic groups and different geographical environmental exposures (Di Pietro et al., 2010), which may explain the divergent results in Brazilians, who form an ethnically heterogeneous population (Carvalho-Silva et al., 2001). It was therefore important to assess polymorphisms in the phase I detoxification enzymes responsible for glutathione conjugation of alkylators and scavenging of free radicals in a population of patients with malignant glioma enrolled in a Phase I/II clinical trial of $\mathrm{POH}$ and who had failed multimodal therapy including surgery, radiation, and chemotherapy (da Fonseca et al., 2008a).

The frequency of GSTM1 deletion in Brazilian adults with malignant gliomas differs from other types of epithelial tumors (Engel et al., 2002; Carlsten et al., 2008; Chen et al., 2010) that show a positive association between GSTM1 deletion and tumor 
development. In addition, a study of 78 patients and 374 controls did not reveal an association between GST polymorphisms and primary glioma risk in Brazilians (Coutinho et al., 2010). In this study, GSTM1 deletion was not significantly correlated $(\mathrm{P}=0.19)$ with the occurrence of malignant glioma in adult Brazilians. GSTM1 deletion was observed in $44 \%$ of glioma patients and $54 \%$ of healthy controls. This result is consistent with a North American study that found a similar association between GSTM1 homozygous deletion and malignant gliomas $(53 \%, \mathrm{OR}=1.1 ; 95 \% \mathrm{CI}=0.7-1.7)$ in 158 patients and 157 controls (Wiencke et al., 1997). Conversely, a significant decrease in the frequency of GSTM1 deletion was observed in pediatric patients with high-grade astrocytoma, but not in adults (Ezer et al., 2002; De Roos et al., 2003; Okcu et al., 2004; Lai et al., 2005; Diedrich et al., 2006; Kilburn et al., 2010).

We also observed GSTT1 deletion in only $11.5 \%$ of patients with malignant gliomas, versus $36 \%$ in healthy controls. This highly significant $(\mathrm{P}=0.00009)$ difference suggests a negative association between GSTT1 gene deletion and the occurrence of malignant gliomas in the Brazilian population in this study. Similarly, GSTT1 deletion has been reported in a low percentage (13 to $16 \%)$ of patients with brain tumors, mostly malignant gliomas (Ezer et al., 2002; Okcu et al., 2004). Interestingly, a study conducted in Rio de Janeiro observed GSTT1 deletion in $25.4 \%$ of 591 healthy volunteers with no history of cancer (Rossini et al., 2002), although GSTT1 deletion may be associated with increased risk of meningioma (Lai et al., 2005).

Genetic polymorphisms in the GST family were associated with survival and toxicity secondary to chemotherapy with nitrosourea alkylating agents in a large group of American Caucasian patients with primary malignant glioma (Okcu et al., 2004; Diedrich et al., 2006). In the present study, GSTM1 or GSTT1 deletion also correlated with reduced survival in glioma patients. Indeed, patients with a GSTM1 deletion had an 8-week reduction in median survival compared to patients without the deletion. Glioma patients with the GSTT1 deletion survived about 10 weeks less than did patients without the deletion.

Established prognostic factors such as patient age, tumor histology, extent of surgical resection, and Karnofsky score often fail to predict survival (Gilbert et al., 2010; Weller et al., 2012). Although being unable to determine the level and localization of GSTP1 protein expression in tumors is considered an important variable associated with survival of malignant glioma patients (Okcu et al., 2004; Diedrich et al., 2006), there is a significant difference $(\mathrm{P}=0.03)$ in survival of patients with lobar gliomas (33 weeks) in comparison to patients with deeply located tumors ( 39 weeks). This is consistent with previous findings that patients with deep gray matter glioma may survive longer than those with tumors in a lobar location (Ramnarayan et al., 2007). The present results partly explain previous data (da Fonseca et al., 2011) showing that tumor location and polymorphisms of GSTMI and GSTT1 may exert additional influence on the survival of patients with malignant gliomas. It is important to conduct larger studies to confirm whether GST genotypes that encode high-activity enzymes influence $\mathrm{POH}$ detoxification, which may be an independent predictor of outcome and response to $\mathrm{POH}$-based therapy.

\section{ACKNOWLEDGMENTS}

Research supported in part by grants from Rio de Janeiro Research Foundation (FAPERJ grants \#E-26/111.263/2010 and \#E-26/110.758/2011) and the National Research Council (CNPq grants \#401943/2010-0 and \#481059/2011-3). 


\section{REFERENCES}

Adamson DC, Rasheed BA, McLendon RE and Bigner DD (2010). Central nervous system. Cancer Biomark. 9: 193-210. Belpomme D, Irigaray P, Hardell L, Clapp R, et al. (2007). The multitude and diversity of environmental carcinogens. Environ. Res. 105: 414-429.

Cabral REC, Cabral Neto JB and Carvalho MGC (2010). Circulating DNA as a biomarker for early detection of cancer: a brief update with an emphasis on lung cancer. Open Lung Cancer J. 3: 38-44.

Carlsten C, Sagoo GS, Frodsham AJ, Burke W, et al. (2008). Glutathione S-transferase M1 (GSTMI) polymorphisms and lung cancer: a literature-based systematic HuGE review and meta-analysis. Am. J. Epidemiol. 167: 759-774.

Carvalho-Silva DR, Santos FR, Rocha J and Pena SD (2001). The phylogeography of Brazilian Y-chromosome lineages. Am. J. Hum. Genet. 68: 281-286.

Chaudhary SC, Alam MS, Siddiqui MS and Athar M (2009). Perillyl alcohol attenuates Ras-ERK signaling to inhibit murine skin inflammation and tumorigenesis. Chem. Biol. Interact. 179: 145-153.

Chen B, Zhou Y, Yang P and Wu XT (2010). Glutathione S-transferase M1 gene polymorphism and gastric cancer risk: an updated analysis. Arch. Med. Res. 41: 558-566.

Coutinho P, Sandim V, Oliveira JA, Alves G, et al. (2010). Lack of association between glutathione S-transferase polymorphisms and primary glioma in a case-control study in Rio de Janeiro. Genet. Mol. Res. 9: 539-544.

da Fonseca CO, Linden R, Futuro D, Gattass CR, et al. (2008a). Ras pathway activation in gliomas: a strategic target for intranasal administration of perillyl alcohol. Arch. Immunol. Ther. Exp. 56: 267-276.

da Fonseca CO, Schwartsmann G, Fischer J, Nagel J, et al. (2008b). Preliminary results from a phase I/II study of perillyl alcohol intranasal administration in adults with recurrent malignant gliomas. Surg. Neurol. 70: 259-266.

da Fonseca CO, Teixeira RM, Ramina R, Kovaleski G, et al. (2011). Case of advanced recurrent glioblastoma successfully treated with monoterpene perillyl alcohol by intranasal administration. J. Cancer Ther. 2: 16-21.

De Roos AJ, Rothman N, Inskip PD, Linet MS, et al. (2003). Genetic polymorphisms in GSTM1, -P1, -T1, and CYP2E1 and the risk of adult brain tumors. Cancer Epidemiol. Biomark. Prev. 12: 14-22.

Di Pietro G, Magno LA and Rios-Santos F (2010). Glutathione S-transferases: an overview in cancer research. Expert. Opin. Drug Metab. Toxicol. 6: 153-170.

Diedrich A, Bock HC, Konig F, Schulz TG, et al. (2006). Expression of glutathione S-transferase T1 (GSTT1) in human brain tumours. Histol. Histopathol. 21: 1199-1207.

Ebrahimkhani S, Asgharian AM, Nourinaier B, Ebrahimkhani K, et al. (2012). Association of GSTM1, GSTT1, GSTP1 and CYP2E1 single nucleotide polymorphisms with colorectal cancer in Iran. Pathol. Oncol. Res. 18: 651-656.

Engel LS, Taioli E, Pfeiffer R, Garcia-Closas M, et al. (2002). Pooled analysis and meta-analysis of glutathione S-transferase M1 and bladder cancer: a HuGE review. Am. J. Epidemiol. 156: 95-109.

Ezer R, Alonso M, Pereira E, Kim M, et al. (2002). Identification of glutathione S-transferase (GST) polymorphisms in brain tumors and association with susceptibility to pediatric astrocytomas. J. Neurooncol. 59: 123-134.

Fleischhacker M and Schmidt B (2007). Circulating nucleic acids (CNAs) and cancer - a survey. Biochim. Biophys. Acta 1775: $181-232$

Gilbert MR, Gonzalez J, Hunter K, Hess K, et al. (2010). A phase I factorial design study of dose-dense temozolomide alone and in combination with thalidomide, isotretinoin, and/or celecoxib as postchemoradiation adjuvant therapy for newly diagnosed glioblastoma. Neuro-Oncol. 12: 1167-1172.

Hayes JD, Flanagan JU and Jowsey IR (2005). Glutathione transferases. Annu. Rev. Pharmacol. Toxicol. 45: 51-88.

Holstein SA and Hohl RJ (2003). Monoterpene regulation of Ras and Ras-related protein expression. J. Lipid. Res. 44: 1209-1215.

Kilburn L, Okcu MF, Wang T, Cao Y, et al. (2010). Glutathione S-transferase polymorphisms are associated with survival in anaplastic glioma patients. Cancer 116: 2242-2249.

Lai R, Crevier L and Thabane L (2005). Genetic polymorphisms of glutathione S-transferases and the risk of adult brain tumors: a meta-analysis. Cancer Epidemiol. Biomark. Prev. 14: 1784-1790.

Lee JM, Wu MT, Lee YC, Yang SY, et al. (2005). Association of GSTP1 polymorphism and survival for esophageal cancer. Clin. Cancer Res. 11: 4749-4753.

Lo HW and Ali-Osman F (2007). Genetic polymorphism and function of glutathione S-transferases in tumor drug resistance. Curr. Opin. Pharmacol. 7: 367-374.

Louis DN, Ohgaki H, Wiestler OD, Cavenee WK, et al. (2007). The 2007 WHO classification of tumours of the central nervous system. Acta Neuropathol. 114: 97-109.

McMartin C, Hutchinson LE, Hyde R and Peters GE (1987). Analysis of structural requirements for the absorption of drugs and macromolecules from the nasal cavity. J. Pharm. Sci. 76: 535-540. 
Ohgaki H and Kleihues P (2005). Epidemiology and etiology of gliomas. Acta Neuropathol. 109: 93-108.

Okcu MF, Selvan M, Wang LE, Stout L, et al. (2004). Glutathione S-transferase polymorphisms and survival in primary malignant glioma. Clin. Cancer Res. 10: 2618-2625.

Pinarbasi H, Silig Y and Gurelik M (2005). Genetic polymorphisms of GSTs and their association with primary brain tumor incidence. Cancer Genet. Cytogenet. 156: 144-149.

Qiu LX, Wang K, Lv FF, Chen ZY, et al. (2011). GSTM1 null allele is a risk factor for gastric cancer development in Asians. Cytokine 55: 122-125.

Ramnarayan R, Dodd S, Das K, Heidecke V, et al. (2007). Overall survival in patients with malignant glioma may be significantly longer with tumors located in deep grey matter. J. Neurol. Sci. 260: 49-56.

Rossini A, Rapozo DC, Amorim LM, Macedo JM, et al. (2002). Frequencies of GSTM1, GSTT1, and GSTP1 polymorphisms in a Brazilian population. Genet. Mol. Res. 1: 233-240.

Schwartzbaum JA, Ahlbom A, Lonn S, Warholm M, et al. (2007). An international case-control study of glutathione transferase and functionally related polymorphisms and risk of primary adult brain tumors. Cancer Epidemiol. Biomark. Prev. 16: 559-565.

Weller M, Stupp R, Hegi M and Wick W (2012). Individualized targeted therapy for glioblastoma: fact or fiction? Cancer J. 18: 40-44.

Wiencke JK, Wrensch MR, Miike R, Zuo Z, et al. (1997). Population-based study of glutathione S-transferase mu gene deletion in adult glioma cases and controls. Carcinogenesis 18: 1431-1433. 